\title{
DIE ERSTEN PROFESSORENGENERATIONEN AN DER KRAKAUER UNIVERSITÄT IM 15. JAHRHUNDERT
}

\author{
KRZYSZTOF OŻÓG
}

\section{THE FIRST GENERATIONS OF PROFESSORS AT THE UNIVERSITY OF KRAKÓW IN THE 15TH CENTURY}

\begin{abstract}
The study presents the first two generations of professors at the University of Kraków in the fifteenth century. The first generation was made up of Polish, Czech, and German professors from the University of Prague, who in the year 1400 organized the university in Kraków. The second generation of professors in Kraków formed thanks to years of work on the part of masters who came to Kraków from Prague and other European universities. Both generations had a decisive influence on the development of the University of Kraków in the fifteenth century.
\end{abstract}

Keywords: university - Kraków - Prague - professor - faculty - generation - fifteenth century

\section{DOI: $10.14712 / 23365730.2020 .15$}

Die Krakauer Universität wurde 1364 von Kasimir dem Großen gegründet. Zu Lebzeiten des Gründers wurden 1370 die Artistenfakultät und die Medizinische Fakultät eröffnet; dagegen ließ sich die Juristenfakultät nicht etablieren. Nach dem Tod von König Kasimir 1370 beendete die Universität ihren Betrieb und die Studenten aus Krakau zogen an die Universität in Prag. Magister, welche die erste Universität in Krakau etabliert haben, sind uns nicht bekannt. ${ }^{1}$ Die Quellen enthalten lediglich Informationen über vier Krakauer Bakkalaurei. Dies waren: Theodorik von Lucca, Nikolaus von Osterhausen, Johannes von Elbing und Nikolaus von Gleiwitz. Drei von ihnen setzten nach dem Bakkalaureat in Krakau ihr Studium in Prag fort. ${ }^{2}$ Der wahrscheinlich einzige, aus den Quellen in Krakau bekannte und im dortigen Studium Generale promovierte Magister war Ambold von Kompina, Rektor der Krakauer Domschule (1355-1371) und Mitarbeiter von Arnald von Caussinh, päpstlicher Kollektor in Polen. ${ }^{3}$ Nach 1370 studierten viele Polen in Prag und absolvierten

1 Stanisław Krzyżanowski, Poselstwo Kazimierza Wielkiego do Awinionu i pierwsze uniwersyteckie przywileje [Die Botschaft Kasimirs des Großen nach Avignon und die ersten Universitätsprivilegien], Rocznik Krakowski 4, 1900, S. 32-45, 60-64 (Nr. 3); Stanisław Szczur, Papież Urban V i powstanie uniwersytetu w Krakowie w 1364 r. [Papst Urban V. und die Entstehung der Universität in Krakau 1364], Kraków 1999, S. 123-216; Krzysztof STOPKA, Głos w dyskusji nad fundacja Uniwersytetu w Krakowie [Ein Wort zur Diskussion der Universitätsgründung in Krakau], Rocznik Krakowski 71, 2005, S. 31-39; Paul W. KnolL, ,A Pearl of Powerful Learning". The University of Cracow in the Fifteenth Century, Leiden - Boston 2016, S. 10-22.

2 S. Szczur, Papież Urban V, S. 204-206.

3 Zofia KozŁowska-Budkowa, W sprawie uniwersytetu kazimierzowskiego (przeglad nowych ustaleń, dyskusji i hipotez) [In Sachen Kasimir-Universität (Übersicht über neue Feststellungen, Diskussionen und Hypothesen)], Studia Historyczne 12, 1969, S. 237-247; hier S. 243-245; Stanisław Kuraś, Amboldus syn Jana 
dort das Studium der freien Künste, des kanonischen Rechts und der Theologie. Manche von ihnen waren für längere Zeit mit den beiden Prager Universitäten verbunden und hielten dort Vorlesungen ab. ${ }^{4}$

Vor 1390 waren vier polnische Gelehrte von Prag nach Krakau gekommen und haben dort 1390 versucht, die Universität zu erneuern: Nikolaus von Gorzków, Doktor der Dekrete, Nikolaus Wigandi von Krakau, Bakkalaureus der Dekrete, Stanislaus von Skalbmierz, Magister Artium, und Bartholomäus von Jasło, Magister Artium. Sie wandten sich an das Königspaar Władysław (Ladislaus) Jagiełło und Hedwig von Anjou mit der Bitte, die Aufzeichnungen von Kasimir dem Großen in der Gründungsurkunde von 1364 zu verwirklichen. Außerdem begannen die erwähnten Professoren, Vorlesungen in Philosophie und Kanonischem Recht abzuhalten. Dies führte zur Initiierung einer Rumpf-Universität, die sich aus drei Fakultäten zusammensetzte: der Freien Künste, des Kirchenrechts und der Medizin. ${ }^{5}$ Im Zusammenhang mit Plänen, eine theologische Fakultät zu gründen, kam 1391 auch der herausragende Magister der Theologie Matthäus von Krakau von Prag in seine Heimatstadt Krakau. Jedoch konnte er diese Pläne nicht umsetzen, weshalb er Krakau nach einigen Monaten wieder verließ. Der Mangel an Entscheidungen des Königspaares hinsichtlich der Professorenbezüge führte dazu, dass die Universität Anfang 1393 erneut zum Erliegen kam. Der König und die Königin beschlossen jedoch, eine neue Universitätsstiftung ins Leben zu rufen, und erreichten bei Papst Bonifatius IX. die Errichtung der Theologischen Fakultät in Krakau, was mit der Bulle vom 11. Januar 1397 geschah. Damals wurde Matthäus von Krakau, der damals bereits Professor für Theologie in Heidelberg war, erneut nach Krakau geladen. Aus unbekannten Gründen konnte er jedoch die Theologische Fakultät nicht organisieren und die Universität Krakau nicht erneuern. Monate später kehrte Matthäus nach Heidelberg zurück. ${ }^{6}$ Im selben Jahr beschloss Königin Hedwig von Anjou, mit Zustimmung des böhmischen Königs Wenzel IV. in Prag ein Kolleg für litauische

z Kęina, pierwszy znany profesor Uniwersytetu Krakowskiego [Ambold von Kepin, der erste bekannte Professor der Universität Krakau], Studia Historyczne 27, 1984, S. 491-493; Krzysztof Ożóg, Intelektualiści w stużbie Królestwa Polskiego w latach 1306-1382 [Intellektuelle im Dienst des Königreichs Polen in den Jahren 1306-1382], Kraków 1996, S. 13, 90, 127.

4 Henryk Barycz, Zwiqzki dziejowe Polski z Uniwersytetem Karola w Pradze [Die historischen Beziehungen Polens zur Karlsuniversität Prag], Przegląd Zachodni 4, 1948, S. 252-269, 339-342; Jadwiga KrzyżAnIAKOwA, Zwiazki uniwersytetu praskiego z Uniwersytetem Krakowskim w drugiej połowie XIV wieku [Die Beziehungen der Prager Universität zur Universität Krakau in der zweiten Hälfte des XIV. Jahrhunderts], AUC-HUCP 5/1-2, 1964, S. 101-132; Dieselbe, Profesorowie krakowscy na uniwersytecie w Pradze - ich mistrzowie $i$ koledzy [Krakauer Professoren an der Universität Prag - ihre Lehrer und Kollegen], in: Waldemar BukowskiKrzysztof Ożóg - Franciszek Sikora (edd.), Cracovia-Polonia-Europa. Studia z dziejów średniowiecza ofiarowane Jerzemu Wyrozumskiemu w sześćdziesiątą piątą rocznicę urodzin i czterdziestolecie pracy naukowej, Kraków 1995, S. 505-527; Josef TŘí̌KA, Repertorium biographicum Universitatis Pragensis praehussiticae 1348-1409, Praha 1981, passim; Krzysztof Ożóg, Uczeni w monarchii Jadwigi Andegaweńskiej i Władysława Jagietty (1384-1434) [Gelehrte in der Monarchie Hedwigs von Anjou und Ladislaus Jagiellos], Kraków 2004, S. 30-33.

5 Maria KowalczyK, Odnowienie Uniwersytetu Krakowskiego w świetle mów Bartłomieja z Jasła [Die Erneuerung der Krakauer Universität im Lichte der Predigten des Bartholomäus von Jaslo], Małopolskie Studia Historyczne 6/3-4, 1964, S. 23-42; Zofia KozŁowskA-Budkowa, Odnowienie jagiellońskie Uniwersytetu Krakowskiego (1390-1414) [Die Erneuerung der jagiellonischen Universität in Krakau (1390-1414)], in: Kazimierz Lepszy (ed.), Dzieje Uniwersytetu Jagiellońskiego w latach 1364-1764, I, Kraków 1964, S. 37-89, hier S. 37-40; P. W. Knoll, , A Pearl of Powerful Learning”, S. 22-28.

6 Z. KozŁowska-Budkowa, Odnowienie jagiellońskie, S. 37-41; Matthias Nuding, Matthäus von Krakau. Theologe, Politiker, Kirchenreformer in Krakau, Prag und Heidelberg zur Zeit des Grossen Abendländischen Schismas, Tübingen 2007, S. 138-141, 144-146 (Spätmittelalter und Reformation, 38). 
Theologiestudenten zu gründen. Mit der Hilfe des Prager Magisters Johannes Štěkna, der seit 1395 mit dem Krakauer Hof verbunden war, war sie um die Gründung bemüht, doch vereitelte ihr Tod 1399 diesen Plan. ${ }^{7}$ Auf ihrem Sterbebett verpflichtete Hedwig Władysław Jagiełło zur erneuten Gründung der Universität und vermachte ihre Juwelen im Wert von rund 2.200 Mark testamentarisch diesem Zweck. -

Am 26. Juli 1400 stellte der König die Gründungsurkunde aus und betraute eine Gruppe von Gelehrten aus Prag, an deren Spitze Stanislaus von Skalbmierz stand, damals schon Doktor der Dekrete und Beichtvater der verstorbenen Königin, mit der Organisation der Universität. Neben Skalbmierz gehörten zu dieser Gruppe: der Doktor der Dekrete Nikolaus von Gorzków, der Doktor der Theologie Johannes Isner, der Bakkalaureus der Theologie (Sententiarius) Johannes Štěkna sowie die Bakkalaurei der Theologie (Biblisten) Bartholomäus von Jasło und Nikolaus Peyser. Alle haben in Prag studiert und gelehrt. ${ }^{8}$ Unter der Leitung von Stanislaus von Skalbmierz, der erster Rektor wurde, entwickelten sie Statuten für die gesamte Universität. Dagegen nahmen in der Kommission, die in den Jahren 1404-1406 die Statuten für die Artistenfakultät festlegten, außer Stanislaus von Skalbmierz die Prager Magister Artium Andreas von Marienburg, Adalbert (Wojciech) von Młodzawy, Erazmus von Neisse und Franciscus von Brieg teil. ${ }^{9}$ Demgegenüber sind uns die Professoren unbekannt, die die ersten Statuten für die Fakultäten für Theologie, Recht und Medizin ausarbeiteten. ${ }^{10}$ Bei der Arbeit an den allgemeinen Universitätsstatuten sowie an denen für die einzelnen Fakultäten nutzten die genannten Magister ihre an den Prager Universitäten gesammelten Erfahrungen. ${ }^{11}$ Eine sehr wichtige Rolle bei der Gründung der Universität von Krakau spielte der Doktor der beiden Rechte Peter Wysz von Radolina, Bischof von Krakau und erster Universitätskanzler, der seinen Doktortitel in Padua erworben hatte. ${ }^{12}$ Im Jahre 1400 wurde die Vorbildnutzung der italienischen Rechtsuniversitäten (Bologna und Padua) in Krakau aufgegeben, doch entstand keine Kopie der Prager Universitäten. König Władysław Jagiełło und seine gelehrten Berater beschlossen, eine Universität mit vier Fakultäten unter der Aufsicht eines Rektors zu gründen, der aus den Reihen der Magister und von ihnen gewählt wurde. Das System der Universitätsnationen, das in den Universitäten vom Typ der Bologneser und Pariser Universität eine sehr wichtige Rolle spielte, wurde in der

7 Celina ZawodzińsKa, Kolegium królowej Jadwigi przy Uniwersytecie Karola w Pradze i jego pierwszy statut [Das Kolleg der Königin Hedwig an der Karlsuniversität und sein erstes Statut], Zeszyty Naukowe Uniwersytetu Jagiellońskiego. Prace historyczne 9, Kraków 1962, S. 19-38; Mlada Holá, Studentské koleje pražské univerzity v pozdním středověku a raném novověku. Dějiny - správa-úřední písemnosti (do roku 1622) [Die Studentenkollegien der Prager Universität im Spätmittelalter und der Frühen Neuzeit. Geschichte - Verwaltung - amtliches Schrifttum (bis 1622)], Praha 2017, S. 93-103.

8 Z. KozŁowska-Budkowa, Odnowienie jagiellońskie, S. 42-49, 56-60; P. W. Knoll, „A Pearl of Powerful Learning", S. 32-38, 79-80.

9 Josephus Muczkowski (ed.), Statuta nec non liber promotionum philosophorum ordinis in Universitate studiorum Jagellonica ab anno 1402 ad annum 1849, Cracoviae 1849, S. I-XXIV; Z. KozŁowsKa-BudKowA, Odnowienie jagiellońskie, S. 56-60, 71,77-82; P. W. KNOLL, „A Pearl of Powerful Learning”, S. 80-81.

10 Z. KozŁowska-Budkowa, Odnowienie jagiellońskie, S. 71.

11 Krzysztof Ożóg, Utrum Universitas Pragensis sit mater Universitatis Cracoviensis? Czyli o wzorcach korporacyjnych krakowskiej wszechnicy $w$ XV wieku [Utrum Universitas Pragensis sit mater Universitatis Cracoviensis? Also über institutionelle Vorbilder der Krakauer Universität im 15. Jahrhundert], in: Wojciech Iwańczak - Janusz Smołucha (edd.), Wspólnoty małe i duże w społeczeństwach Czech i Polski w średniowieczu i w czasach nowożytnych, Kraków 2010, S. 63-78.

12 Mieczysław Gębarowicz, Psalterz floriański i jego geneza [Der Florianer Psalter und seine Genese], Wrocław 1965, S. 43-84. 
Universität von Krakau nicht eingeführt. In ihrer Struktur können zahlreiche Ähnlichkeiten mit der Dreifakultätenuniversität in Prag festgestellt werden. ${ }^{13}$

Den Organisatoren der zweiten Universität in Krakau gelang es, mehrere Dutzend Magister aus Prag zu gewinnen, was durch zwei Professorenlisten aus den Jahren 1404/1405 und 1408 bestätigt wird. ${ }^{14}$ Zwar waren es hauptsächlich polnische Gelehrte, doch befanden sich unter ihnen auch tschechische und deutsche Magister. Eine große Gruppe von Gelehrten kam in den Jahren 1400-1404/1405 nach Krakau. Insgesamt gab es 31 Professoren, darunter 22 Artisten, 4 Kanonisten, 4 Theologen und 1 Mediziner. ${ }^{15}$ Neben den oben Genannten waren es die Magister Artium Johannes Silvanus von Prag, Fredericus Zuner von Rietheim, Stefan Hopper, Johannes von Kreuzburg, Jakob von Biecz, Antonius Tempelfeld von Krakau, Stanislaus von Żarnowiec, Pełka von Borzyków, Nikolaus von Arnschwang, Johannes von Fałków, Gregorius Benedicti von Krakau, Nikolaus von Kozłów, Andreas von Kokorzyn, Wilhelm Kesinger von Gundelfingen, Matthias von Koło, Nikolaus Sculteti von Konradswaldau, Peter von Brandys und Elias von Wąwolnica, ferner der Lizentiat der Dekrete, Stefan Mladota von Czersk, der Magister der Medizin Thomas von Amelia sowie die Bakkalaurei der Medizin Heinrich Alman und Andreas Pyrnycer. ${ }^{16}$ Außerdem lehrte zu dieser Zeit an der Krakauer Universität auch der Doktor der Dekrete Nikolaus Wigandi von Krakau, der gleichzeitig Theologie studierte. ${ }^{17}$

Der Zustrom von Magistern aus Prag nach Krakau setzte sich weiter fort, was in dem Professorenverzeichnis von 1408 dokumentiert ist. In ihm fallen die neuen Prager Gelehrten ins Auge: der Doktor der Theologie Mauritius Rvačka, die Magister Artium Johannes Wilusz von Krakau und Heinrich von Kłobuck. ${ }^{18}$ In diesem Verzeichnis treten Magister Artium auf, die das Bakkalaureat noch an der Prager Universität erworben hatten und ihr Studium dann in Krakau fortsetzten, wo sie den Grad eines Magisters erwarben und Philosophievorlesungen hielten. Zu dieser Gruppe gehörten: Nikolaus von Glogau, Budislaus von Krakau, Lukas von Wielki Koźmin, Johannes Supparii, Heinrich von Rosenberg und vermutlich auch Nikolaus Hinczowic von Kazimierz und Nikolaus von Bieganów, die 1403 in Krakau den Magistergrad in den Freien Künsten erwarben. ${ }^{19}$ Wir haben keine Informationen über ihre Bakkalaureate im Prager Liber decanorum und im Krakauer Liber promotionum. Im Verzeichnis fehlt auch Andreas Wężyk von Giebułtów, der es 1397 in Prag zum Bakkalaureus der Freien Künste gebracht hatte und 1402 in Krakau den Magistergrad erwarb. Jahre später war er wieder an der Prager Universität, wo er in den Jahren

13 K. Ożóg, Utrum Universitas Pragensis, S. 67-80.

14 Antoni GĄSIOROwSKi - Tomasz JuREK - Izabela SkIERSKA - Ryszard GrzesiK (edd.), Metryka Uniwersytetu Krakowskiego z lat 1400-1508. BJ rkp. 258, I-II [Die Matrikel der Universität Krakau aus den Jahren 1400-1508. BJ Ms. 258, I-II], Kraków 2004, I, S. 24-28; Antoni GĄsiorowsKi - Tomasz JureK - Izabela Skierska (edd.), Najstarsza księga promocji Wydziału sztuk Uniwersytetu Krakowskiego z lat 1402-1541 [Das älteste Promotionsbuch der Artistenfakultät der Universität Krakau aus den Jahren 1402-1541], Warszawa 2011, S. 192-193; Z. KozŁowsKa-BudKowa, Odnowienie jagiellońskie, S. 60-69.

15 Krzysztof Ożóg, Migrationen von Professoren und Studenten aus Prag nach Krakau zu Beginn des 15. Jahrhunderts, AUC-HUCP 49/2, 2009, S. 99-112, hier S. 102-103.

16 A. GąSIOROwSKi - T. JureK - I. SKIERSKA - R. GrZeSIK (edd.), Metryka Uniwersytetu, I, S. 25-28.

17 Z. KozŁowska-Budkowa, Odnowienie jagiellońskie, S. 65.

18 A. Gąsiorowski - T. JureK - I. Skierska (edd.), Najstarsza księga promocji, S. 192-193.

19 A. GĄSIOROWSKi - T. JUREK - I. SKIERSKA (edd.), Najstarsza księga promocji, S. 194-195. 
1408-1409 an der Artistenfakultät Prüfungen abnahm..$^{20}$ Es ist nicht bekannt, wo Laurentius von Uppsala, der in dem Professorenverzeichnis von 1408 steht, seinen Magistergrad erworben hatte. ${ }^{21}$ Der oben besprochenen Professorengruppe schlossen sich schnell auch die ersten Magister Artium an, die ihr ganzes Studium an der Krakauer Artistenfakultät absolvierten. Im Jahre 1408 waren dies: Nikolaus Budziszyn von Krakau, Jarosław von Służów, Jakob von Kralovice, Johannes von Jasiona, Wilhelm von Mogiła und Nikolaus von Bidziny. ${ }^{22}$ Insgesamt hielten 1405 in Krakau 32 Professoren Vorlesungen, 1408 waren es demgegenüber 41. Einige Gelehrte verließen Krakau recht schnell. Für kurze Zeit kehrten Peter von Brandys und Mauritius Rvačka 1408 nach Prag zurück, Heinrich von Kłobuck 1409 hingegen für immer. ${ }^{23}$ Thomas von Amelia, Heinrich Alman, Fredericus Zuner von Rietheim und Nikolaus von Arnschwang fehlen nach 1405 in den Krakauer Quellen und nach 1408 auch Laurentius von Uppsala und Andreas von Doren. Im ersten Jahrzehnt des 15. Jh. kamen 41 Wissenschaftler von Prag nach Krakau. Sie bildeten den Kern der ersten Magister- und Bakkalaureus-Generation in allen vier Fakultäten der Krakauer Universität. Diese Gruppe dominierten Gelehrte aus der polnischen Nation der Prager Universitäten, die aus dem Königreich Polen und Schlesien stammten. Doch gab es auch Magister, die aus anderen als der polnischen Universitätsnation kamen: aus der bayerischen Friedrich Zuner, Nikolaus von Arnschwang und Wilhelm Kesinger, aus der sächsischen Stefan Hopper, aus der böhmischen Nation Johannes Štěkna, Peter von Brandys, Johannes Silvanus von Prag und Mauritius Rvačka. Im zweiten Jahrzehnt des 15. Jahrhunderts kamen noch von Prag nach Krakau, und zwar entweder über Leipzig und Wien oder auf direktem Wege, zwei Doktoren der Medizin: Vincentius Viaw von Schweidnitz und Johannes Kro von Cottbus, zwei Doktoren der Theologie: Mathias von Liegnitz und Johannes Frankenstein, sowie sechs Magister Artium: Augustinus von Bardejov, Eberhard Hatfeld, Paulus von Wurzen, Olaf von Uppsala, Augustinus von Münsterberg und Johannes Sneschwitz von Breslau. ${ }^{24}$ Insgesamt zogen gegen Ende des 14. Jahrhunderts und der ersten beiden Jahrzehnte des 15. Jahrhunderts nach Krakau 54 Bakkalaurei, Magister, Lizentiaten und Doktoren, die die intellektuellen Errungenschaften Prags in das Universitätsmilieu Krakaus implementierten. ${ }^{25}$

Die zweite Krakauer Professorengeneration wuchs infolge einer mehrjährigen Tätigkeit von Magistern heran, die von Prag und anderen europäischen Universitäten nach Krakau gekommen waren. Dies lässt sich am besten anhand einer Analyse der Zusammensetzung der Professoren erkennen, die an bestimmten Fakultäten unterrichteten. An der personenreichsten Artistenfakultät bestand in den ersten beiden Jahrzehnten des 15. Jahrhunderts der Kern der Lehrbeauftragten aus Magistern, die von der Universität Prag stammten. Davon zeugt die Besetzung des Dekan-Amtes dieser Fakultät. Die Funktion des Dekans wurde zu

20 A. Gąsiorowski - T. JureK - I. Skierska (edd.), Najstarsza księga promocji, S. 194; J. TŘišKa, Repertorium biographicum, S. 30.

21 A. GĄSIOROWSKI - T. JUREK - I. SKIERSKa (edd.), Najstarsza księga promocji, S. 193.

22 A. GąsiorowsKi - T. JUREK - I. Skierska (edd.), Najstarsza księga promocji, S. 194-196.

23 J. Tří̌̌Ka, Repertorium biographicum, S. 158, 443; Mieczysław Markowsкi, Dzieje Wydziału Teologii Uniwersytetu Krakowskiego w latach 1397-1525 [Geschichte der Theologischen Fakultät der Krakauer Universität in den Jahren 1397-1525], Kraków 1996, S. 108-109.

24 A. Gąsiorowski - T. Jurek - I. Skierska - R. Grzesik (edd.), Metryka Uniwersytetu, I, S. 28-29; K. Ożóg, Migrationen von Professoren, S. 108-111.

25 K. Ożóg, Migrationen von Professoren, S. 111-112. 
dieser Zeit mehrfach wahrgenommen von Franciscus von Brieg, Andreas von Marienburg, Nikolaus Sculteti von Konradswaldau, Peter von Brandys, Andreas von Kokorzyn, Pełka von Borzyków, Johannes Wilusz von Krakau. Die letzten Dekane der zugereisten Magister waren Augustinus von Münsterberg (im Wintersemester 1417/1418), Olaf von Uppsala (im Sommersemester 1418 und höchstwahrscheinlich im Wintersemester 1418/1419), Paulus von Wurzen (im Wintersemester 1419/1420) sowie Peter von Brandys (im Wintersemester 1422/1423) ${ }^{26}$ Nach fünfzehnjährigem Betrieb der Krakauer Universität war im Wintersemester 1414/1415 und im Sommersemester 1415 einer ihrer ersten Absolventen, Nikolaus Budziszyn von Krakau (1400 immatrikuliert, Bakkalaureus Artium 1402 und Magister Artium ab 1404), Dekan der Artistenfakultät. Die folgenden Dekane, die ihre Grade im Bereich der Freien Künste ausschließlich in Krakau erwarben, waren Jakob von Jasło, Johannes von Radochońce, Peter von Sienno, Nikolaus von Piątek, Stanislaus von Sobniów, Stanislaus von Piotrków, Benedikt Hesse, Laurentius von Ratibor, Nikolaus von Oszkowice, Andreas von Buk, Sigismund von Pyzdry, Johannes Orient, Nikolaus Tempelfeld von Brieg, Johannes von Jastrzębie, Johannes Czelp von Wieluń, Paulus von Pyskowice und Stanislaus von Przechowo. ${ }^{27}$ Sie setzten die Arbeit der ersten Professorengeneration auf dem Gebiet der Lehre und Weiterentwicklung der aristotelischen Philosophie an der Krakauer Universität fort. ${ }^{28}$

Die Gelehrten, die im Jahr 1400 die Universität in Krakau etablierten, legten im 15. Jahrhundert den Grundstein für ihre geistige Entwicklung. Im Rahmen des Lehrplans der Freien Künste an der Krakauer Universität im 15. Jahrhundert wurde ein universelles Programm umgesetzt, dessen Grundlagen an der Pariser Universität entwickelt und in reifer Form nach Prag und von dort nach Krakau verpflanzt wurde..$^{29}$ Das intellektuelle Bild der Artistenfakultät, das sich den beiden Generationen der Krakauer Magister verdankte, zeigt, dass die kreative Errungenschaft der europäischen Wissenschaft auf dem Gebiet der Philosophie und der exakten Wissenschaften an der Krakauer Universität ihre Heimstatt hatte. Kommentare zu bestimmten Werken von Aristoteles und anderen Autoren, die an zeitgenössischen Artistenfakultäten europäischer Universitäten gelehrt wurden, brachte man nach Krakau zumeist aus Prag. ${ }^{30}$ Die Kommentare, die im Krakauer Milieu benutzt wurden, repräsentierten verschiedene doktrinäre Strömungen. Auch standen die Krakauer Magister, sofern sie ihre eigenen Kommentare verfassten, den Meinungen von Gelehrten der via moderna

26 A. GĄSIOROWSKi - T. JuREK - I. SkIERSKa (edd.), Najstarsza księga promocji, S. 194-202, Antoni GąsiorowSKI, Bylina i inni. Dziekani Wydziału Sztuk krakowskiego uniwersytetu w XV wieku [Bylina und andere. Die Dekane der Artistenfakultät der Krakauer Universität im 15. Jahrhundert], in: Wojciech Iwańczak - Stefan K. Kuczyński (edd.), Ludzie, Kościół, wierzenia. Studia z dziejów kultury i społeczeństwa Europy Środkowej (średniowiecze - wczesna epoka nowożytna), Warszawa 2001, S. 523-537, hier S. 525-526, 532-533.

27 A. Gąsiorowski - T. JureK - I. Skierska (edd.), Najstarsza księga promocji, S. 198-207; A. GĄSIOROwski, Bylina i inni, S. 526, 532-533.

28 K. OżóG, Uczeni w monarchii, S. 45-52.

29 František Šmahel, The Faculty of Liberal Arts, in: Ivana Čornejová - Michal Svatoš, A History of Charles University, I, A History of Charles University 1348-1802, Prague 2001, S. 93-114; Krzysztof OżóG, Zakres i metody nauczania septem artes na Wydziale Sztuk Uniwersytetu Krakowskiego w XV wieku [Fach und Lehrmethoden der Septem Artes an der Fakultät der Freien Künste der Universität Krakau im 15. Jahrhundert], in: Teresa Michałowska (ed.), Septem artes w kształtowaniu kultury umysłowej w Polsce średniowiecznej (wybrane zagadnienia), Wrocław 2007, S. 105-111.

30 Krzysztof Ożóg, The Role of Poland in the Intellectual Development of Europe in the Middle Ages, Kraków 2009, S. 94-100. 
und antiqua gegenüber offen, allerdings mit Überlegenheit ersterer. ${ }^{31}$ In den ersten drei Jahrzehnten des 15. Jahrhunderts wurde für die Krakauer Schule der Moralphilosophie, der Philosophie des Seins und der Naturphilosophie sowie der Astronomie eine solide Grundlage gelegt.

An der Krakauer Juristenfakultät wurden nach der zweiten Universitätsstiftung nur Vorlesungen über kanonisches Recht gehalten. Das gesamte Corpus iuris canonici (Decretum von Gracjan, Decretales von Gregor IX., Liber Sextus von Bonifatius VIII. und Clementinae) wurde behandelt. Zu den ersten Professoren dieser Fakultät gehörten die Prager Doktoren der Dekrete, d.h. Nikolaus von Gorzków und Stanislaus von Skarbimierz, sowie der Bakkalaureus der Dekrete Stefan Mladota. Im Laufe der Zeit hielt um 1407 auch der Doktor der Dekrete Nikolaus Wigandi Vorlesungen an dieser Fakultät. ${ }^{32}$ In den darauffolgenden Jahren wurde die Fakultät durch den in Prag und Padua ausgebildeten Doktor der Dekrete Paulus Wladimiri unterstützt (seine Promotion fand mit Zustimmung des Pisa-Papstes Johannes XXIII. 1411 in Krakau statt). Außerdem wurde Peter Wolfram, Lizentiat der Dekrete, nach seinem Studium in Prag, Padua und Bologna 1413 Professor für Kanonisches Recht in Krakau..$^{33}$ Aus der obigen Aufstellung ergibt sich, dass die Krakauer Juristenfakultät zu Beginn ihres Bestehens auf den in Prag, Padua und Bologna ausgebildeten Gelehrten ruhte. Einen wesentlichen Einfluss auf die doktrinären Grundlagen der Krakauer Kanonistik hatte Peter Wysz, Doktor beider Rechte, der als Universitätskanzler seine Tätigkeit am 26. Juli 1400 mit einem Vortrag aus den Dekretalen (Decretales) Gregors IX. eröffnete. ${ }^{34}$ Dagegen wies Stanislaus von Skarbimierz in seiner Eröffnungsrede als Rektor der Juristenfakultät eine wichtige Rolle zu. Seiner Meinung nach führt das Kanonische Recht als Wissenschaft, die sich mit spirituellen und säkularen Dingen befasst, den Menschen zur Perfektion und hat universellen Charakter. Darüber hinaus behandelte er die Lehre von Recht und Theologie gleichermaßen, indem er die Doktoren der Dekrete und der Theologie als zwei Säulen der Kirche anerkannte. ${ }^{35}$

Bereits im ersten Jahrzehnt des 15. Jahrhunderts wurden in Krakau die Absolventen der Prager Artistenfakultät zu Bakkalaurei der Dekrete gefördert: Johannes von Fałków (1407), Adalbert (Wojciech) von Młodzawy (1408) und Mathias von Koło (um 1408). Im Laufe der Zeit erwarben sie die Lizentiate sowie Doktorgrade im kanonischen Recht, und so wurde Johannes von Fałków 1418 Doktor der Dekrete, Mathias von Koło hingegen 1423 Lizentiat. Diese Gelehrten waren der Ansatz zur zweiten Generation von Kanonisten an der Krakauer Universität. ${ }^{36} \mathrm{Ihr}$ schlossen sich auch die Doktoren der Dekrete an, die nicht nur in Krakau, sondern auch an anderen europäischen Universitäten ihren Abschluss gemacht hatten. Dazu gehörte Władysław von Oporów, der zunächst an der Universität Wien die Artes Liberales studierte (1415 erwarb er dort den Grad des Bakkalaureus) und

31 Krzysztof OżóG, Uniwersytet Krakowski: przykład późnośredniowiecznego eklektyzmu [Die Krakauer Universität: ein Beispiel spätmittelalterlichen Eklektizismus'], in: Teresa Wolińska - Mirosław J. Leszka (edd.), Średniowieczna wizja świata - jedność czy różnorodność. Idee i teksty, Łódź 2009, S. 235-250, hier S. 240-244.

32 Z. KozŁowska-Budkowa, Odnowienie jagiellońskie, S. 57-58, 65-67; K. Ożóg, The Role of Poland, S. 103-104.

33 Jan FIJAEEK, Polonia apud Italos scholastica saeculum XV, Cracoviae 1900, S. 3-9, 35-44.

34 Metryka Uniwersytetu Krakowskiego, I, S. 11; M. GĘBArowicz, Psalterz floriański, S. 53-56.

35 Juliusz Domański, Discours d'inauguration fait par Stanislas de Skarbimierz à l'occasion du renouveau de l'Université de Cracovie, Mediaevalia Philosophica Polonorum 24, 1979, S. 125-129.

36 Z. KozŁowska-Budkowa, Odnowienie jagiellońskie, S. 66-67. 
dann ab 1416 Kanonisches Recht in Bologna. Nach zwei Jahren zog er nach Padua, wo er 1419 den Doktorgrad der Dekrete erwarb. Nach seiner Rückkehr nach Polen 1420 wurde er in das Professorenverzeichnis der Krakauer Universität aufgenommen und unterrichtete Kanonisches Recht. ${ }^{37}$ Adam von Będków wurde 1423 in Bologna zum Doktor der Dekrete befördert. Danach kehrte er nach Krakau zurück, wo er seit 1425 Professor für Kanonisches Recht war. ${ }^{38}$ Adalbert (Wojciech) Jastrzębiec promovierte 1424 in Bologna im Kanonischen Recht und unterrichtete dann Dekrete in Krakau. ${ }^{39}$ Im dritten und frühen vierten Jahrzehnt des 15. Jahrhunderts wuchs die Gruppe der Kanonisten um weitere Doktoren an, die an der Juristenfakultät in Krakau promoviert hatten. Es waren dies Jakob von Zaborów (1420), Nikolaus Hinczowic von Kazimierz (1422), Thomas von Chrobrze (1423), Nikolaus von Błonie (um 1427), Johannes Elgot (1427), Derslaus von Borzymów (1430), Paulus von Zator (1430), Thomas Strzępiński (1431), Michael von Szydłów (1433), Stanislaus von Ujście (1434) und Martin von Holeszów (um 1435). ${ }^{40}$ Gerade sie bildeten den Kern der zweiten Professorengeneration an der Juristenfakultät der Krakauer Universität.

An der Juristenfakultät nahmen die berühmten Kanonisten Stanislaus von Skarbimierz und Paulus Wladimiri eine wissenschaftliche Reflexion über das Völkerrecht (ius gentium) und die Macht des Papstes und Kaisers über die Heiden vor. Sie interessierten sich besonders für die Beziehungen zwischen Christen und Heiden. ${ }^{41}$ Darüber hinaus befassten sich die Krakauer Professoren an dieser Fakultät (u.a. Stanislaus von Skarbimierz, Jakob von Zaborów, Derslaus von Borzymów und Johannes Elgot) mit der Lösung schwieriger Fälle, die die Beichte und Buße, die Liturgie und die Teilnahme der Gläubigen am Kult, die Familienethik, das Sakrament der Eucharistie sowie die wirtschaftlichen und sittlichen Fragen betrafen. Ihr Engagement galt auch der legislativen Tätigkeit der polnischen Kirche auf Provinz- und Diözesansynoden. ${ }^{42}$

Die Medizinische Fakultät nahm ihren Betrieb schon 1400 auf. Im Kreis der ersten Professoren dieser Fakultät finden sich der Magister für Medizin Thomas von Amelia sowie zwei Medizinbakkalaurei, Heinrich Alman und Andreas Pyrnycer. Im Jahre 1407 schrieb sich Nikolaus von Kayrt aus Ungarn, Bakkalaureus der Medizin, in die Matrikel der Universität Krakau ein. Die Genannten wirkten jedoch nur für kurze Zeit in Krakau. ${ }^{43}$ Erst in

37 A. Gąsiorowski - T. Jurek - I. Skierska - R. Grzesik (edd.), Metryka Uniwersytetu, I, S. 31; Krzysztof OżóG, Władysław z Oporowa h. Sulima (zm. 11 III 1453 r.) [Ladislaus von Oporow der Wappengemeinschaft Sulima (gest. 11.3.1453)], in: Wacław Uruszczak (ed.), Profesorowie Wydziału Prawa Uniwersytetu Jagiellońskiego, I, 1364-1780, Kraków 2015, S. 434-435.

38 A. Gąsiorowski - T. Jurek - I. Skierska - R. Grzesik (edd.), Metryka Uniwersytetu, I, S. 32; J. FijaŁek, Polonia apud Italos, S. 62-65.

39 J. Fijalek, Polonia apud Italos, S. 70-73.

40 A. Gąsiorowski - T. Jurek - I. Skierska - R. Grzesik (edd.), Metryka Uniwersytetu, I, S. 29-34; K. OżóG, Uczeni w monarchii, S. 55.

41 K. OżóG, The Role of Poland, S. 111-119; Wojciech Świeboda, Innowiercy w opiniach prawnych uczonych polskich $w$ XV wieku. Poganie, żydzi, muzutmanie [Andersgläubige nach den Meinungen polnischer Juristen im 15. Jahrhundert. Heiden, Juden, Muslime], Kraków 2013, S. 141-160, 175-213.

42 Krzysztof Ożóg, Prawo kościelne w Polsce XIII-XV stuleciu [Das Kirchenrecht im Polen des 13.-15. Jahrhunderts], in: Pavel Krafl (ed.), Sacri canones servandi sunt. Ius canonicum et status ecclesiae saeculis XIII-XV, Praha 2008, S. 57-80, hier S. 70-72; Krzysztof BRACHA, Casus pulchri de vitandis erroribus conscientiae purae. Orzeczenia kazuistyczne kanonistów i teologów krakowskich wXV wieku [Kasuistische Sentenzen der Krakauer Kanonisten und Theologen im 15. Jahrhundert], Warszawa 2013, S. 26-56.

43 A. Gąsiorowski - T. JureK - I. Skierska - R. Grzesik (edd.), Metryka Uniwersytetu, I, S. 26-27, 63 [07/010]; Z. KozŁowska-Budkowa, Odnowienie jagiellońskie, S. 61. 
der zweiten Dekade des fünfzehnten Jahrhunderts konnte die Fakultät verstärkt werden, da um 1416 Vincentius von Schweidnitz, Doktor der Medizin, und Johannes Kro von Cottbus (im Februar 1416 in Wien zum Doktor der Medizin promoviert) sowie Monaldus von Lucca um 1416 Professoren der Fakultät wurden. ${ }^{44}$ Etwa 1420, vielleicht auch viel früher, begann der Mediziner Johannes de Saccis von Pavia, der wahrscheinlich bereits Ende der neunziger Jahre des 14. Jahrhunderts mit dem Hof von Hedwig und Władysław Jagiełło verbunden war, mit Vorlesungen an der Krakauer Universität. ${ }^{45}$ Der erwähnte Johannes Kro bekleidete im Wintersemester 1419/1420 das Amt des Rektors der Krakauer Universität, während Johannes de Saccis 1425 während der in Krakau herrschenden Pest dieses Amt bekleidete. ${ }^{46}$ Diese Wissenschaftler bildeten die zweite Professorengeneration der Medizinischen Fakultät aus, die von Nikolaus Oszkowski, Johannes von Dobra, Hermann von Przeworsk, Johannes von Ludzisko und Bernard Hesse gebildet wurde. ${ }^{47}$ Einige von ihnen ergänzten ihr Medizinstudium in Italien und promovierten dort. Auch in Krakau erfolgten Promotionen zum Doktor der Medizin. So erlangte um 1430 Hermann von Przeworsk den Doktortitel. ${ }^{48}$ Johannes von Dobra als Krakauer Medizinlizentiat promovierte hingegen vermutlich 1433 an der Universität Padua. Im selben Jahr promovierte hier auch Johannes von Ludzisko. ${ }^{49}$ Die sich entfaltende Fakultät wurde 1433 durch den damaligen Dekan Johannes de Saccis von Pavia reformiert, der ihre Statuten ausarbeitete. Die Krakauer Medizin stand in engem wissenschaftlichen Kontakt mit den größten europäischen Zentren für medizinische Studien, vor allem in Padua. Davon zeugen nicht nur die Doktorgrade, die von den Krakauer Magistern in Padua erworben wurden, sondern auch ihre Büchersammlungen. ${ }^{50}$

Die Grundlagen der Theologischen Fakultät in Krakau wurden von folgenden Gelehrten gelegt: Johannes Isner, Johannes Štěkna und Mauritius Rvačka. Sie haben in Krakau das Theologische Studienprogramm von Prag implantiert und hielten ab 1400 Vorlesungen in Theologie ab. Zur selben Zeit kamen Magister der Freien Künste von Prag nach Krakau; sie hatten ihr Theologiestudium noch an der Universität Prag begonnen oder es schon an der Krakauer Universität aufgenommen. Zu ihnen gehörte Bartholomäus von Jasło, Magister Artium und biblischer Bakkalaureus, denn um 1404 wurde er Sententiarius und zwei Jahre später Doktor der Theologie. ${ }^{51}$ An der Krakauer Universität promovierte 1404 Johannes Silvanus von Prag (ab 1394 in Polen als Beichtvater und königlicher Prediger anwesend)

44 A. Gąsiorowski - T. Jurek - I. Skierska - R. Grzesik (edd.), Metryka Uniwersytetu, I, S. $29,31$.

45 A. Gąsiorowski - T. JureK - I. Skierska - R. Grzesik (edd.), Metryka Uniwersytetu, I, S. 29; K. Ożóg, Uczeni w monarchii, S. 53, 322 .

46 A. Gąsiorowski - T. JureK - I. Skierska - R. Grzesik (edd.), Metryka Uniwersytetu, I, S. 100, 124; Aleksander Birkenmajer, Jan de Saccis, in: Polski słownik biograficzny, IX, Wrocław 1962-1964, S. 473-475.

47 A. Gąsiorowski - T. Jurek - I. Skierska - R. Grzesik (edd.), Metryka Uniwersytetu, I, S. 32-33; K. OżóG, Uczeni w monarchii, S. 53.

48 Aleksander Birkenmajer, Herman z Przeworska [Herman von Przeworsk], in: Polski słownik biograficzny, IX, Wrocław 1960-1961, S. 461-462; Maria KowALCZYK, Lekarz krakowski Jan z Dobrej [Der Krakauer Arzt Johannes von Dobra], Studia Mediewistyczne 34/35, 2000, S. 260-283, hier S. 261-263.

49 J. FijaŁek, Polonia apud Italos, S. 79-83; M. KowalczyK, Lekarz krakowski, S. 261-263.

50 Mieczysław Skulimowski, Pierwsze ustawy Wydziału Lekarskiego Uniwersytetu Krakowskiego z r. 1433 [Das erste Statut der Medizinischen Fakultät der Universität Krakau von 1433], Archiwum Historii Medycyny 23, 1960, S. 221-234; M. KowALCZYK, Lekarz krakowski, S. 266-273, 278-282.

51 Maria KowalczyK, Bartłomiej z Jasła [Bartholomäus von Jaslo], Materiały i Studia Zakładu Historii Filozofii Starożytnej i Średniowiecznej 5, 1965, s. 3-23, hier S. 3-6; M. Markowski, Dzieje Wydziału, S. $104-105$. 
zum Doktor der Theologie, die er allerdings nur bis 1408 unterrichtete..$^{52}$ Unmittelbar nach ihm wurde Nikolaus von Pyzdry promoviert (1406 oder 1407), und 1409 krönte Franciscus von Brieg sein Studium mit dem Doktorgrad. ${ }^{53}$ Mit der Krakauer Universität verbunden war nach 1409 der Prager Doktor der Theologie Mathias von Liegnitz, älterer Bruder von Johannes von Kreuzburg. Seine Tätigkeit in Krakau war nur von kurzer Zeit, da der Gelehrte um 1413 starb. ${ }^{54}$ Trotz des Todes von Bartholomäus von Jasło (ca. 1407), Johannes Štěkna (1407) und Johannes Isner (1411) konnte die Theologische Fakultät dank der in Krakau im ersten und Anfang des zweiten Jahrzehnts des 15. Jahrhunderts promovierten Bakkalaurei und Doktoren nach wie vor normal funktionieren. Neben den oben genannten schlossen sich in dieser Zeit Elias von Wąwolnica (Doktor vermutlich 1412), Andreas von Marienburg (Doktor vermutlich 1413), Erasmus von Neiße (Doktor um 1413) und Lucas von Wielki Koźmin (biblischer Bakkalaureus 1410) zum Theologenkreis zusammen. Diese Gelehrtengruppe war die erste Professorengeneration der Theologischen Fakultät. ${ }^{55}$ Sie bildeten die nächste Generation von Theologen aus, zu der Jakob von Nowy Sącz (Doktor 1421/1422), Johannes von Kreuzburg (Doktor 1423), Andreas von Kokorzyn (Doktor 1425), Nikolaus von Kozłów (Doktor 1425), Sigismund von Pyzdry (Sententiarius 1426), Benedikt Hesse (Doktor 1431), Nikolaus Tempelfeld von Brieg (Bakkalaureus der Theologie 1428), Nikolaus Budziszyn (Doktor 1422), Andreas von Buk (biblischer Bakkalaureus 1427/1428), Jakob von Paradies (Doktor 1432) und Laurentius von Ratibor (Doktor 1433) gerechnet werden können. ${ }^{56}$

Die Werke der Krakauer Theologen des fünfzehnten Jahrhunderts haben einen eklektischen Charakter. Sie waren durch eine konkordistische Haltung gekennzeichnet, während sie in der Methodologie viam communem repräsentierten und Kompromisslösungen in grundlegenden Angelegenheiten suchten. ${ }^{57}$ Das Schaffen der Krakauer Theologen drückte sich in den Kommentaren zu den Sentenzen von Petrus Lombardus, in Bibelkommentaren, Predigten, liturgischen Traktaten, Quaestionen und Traktaten verschiedener Art aus. Viele von ihnen griffen wichtige Themen der damaligen Zeit auf, wie das Problem des Hussitismus, die Reform der Kirche, die Sakramente, mariologische und moralpraktische Fragen. ${ }^{58}$

Die beiden ersten Professorengenerationen beeinflussten maßgeblich die Entwicklung der Krakauer Universität im 15. Jahrhundert. Die erste Generation, die sich hauptsächlich aus dem Prager Milieu rekrutierte, organisierte in Krakau eine Universität mit vier Fakultäten, die 1400 von Władysław Jagiełło gestiftet wurde. Außerdem hat sie an der Krakauer Universität die intellektuellen Errungenschaften der lateinischen Welt eingeführt. Die zweite Professorengeneration, die sich bereits in Krakau herausbildete, richtete ihre wissenschaftliche Reflexion hauptsächlich auf Natur-, Moral- und Daseinsphilosophie,

52 J. TŘišKa, Repertorium biographicum, S. 193; M. Markowski, Dzieje Wydziału, S. 107-108; K. Ożóg, Uczeni w monarchii, S. 311-312.

53 J. TŘí̌̌Ka,, Repertorium biographicum, S. 103, 411; M. Markowski, Dzieje Wydziału, S. 109-110,112-113.

54 J. Tří̌̌Ka,, Repertorium biographicum, S. 364; M. Markowski, Dzieje Wydziału, S.108.

55 M. Markowski, Dzieje Wydziału, S. 113-117.

56 M. Markowski, Dzieje Wydziału, S. 122-148.

57 Zofia WŁodeK, Tendencje doktrynalne na Wydziale Teologicznym Uniwersytetu Krakowskiego w XV wieku [Die doktrinalen Richtungen an der Theologischen Fakultät der Krakauer Universität im 15. Jahrhundert], in: Teresa Michałowska (ed.), Literatura i kultura późnego średniowiecza w Polsce, Warszawa 1993, S. 17-28, hier S. 23-27; M. Markowski, Dzieje Wydziału, S. 118-119; K. OżóG, Uniwersytet Krakowski, S. 244-246.

58 Z. WŁodek, Tendencje doktrynalne, S. 18-21; M. Markowski, Dzieje Wydziału, S. 100-148; P. W. Knoll, "A Pearl of Powerful Learning”, S. 470-536. 
Astronomie und Völkerrecht (ius gentium), im Bereich der Theologie hingegen auf Fragen der Reformen der Kirche in capite et in membris, auf Sakramente, Hussitismus sowie auf moralpraktische Fragen. Dank dieser beiden Magistergenerationen wurde die Krakauer Universität zu einem der wichtigsten intellektuellen Zentren in Mittel- und Osteuropa und zog in der zweiten Hälfte des 15. und Anfang des 16. Jahrhunderts viele Studenten von außerhalb des Königreichs Polen an.

(Sprachliche Korrektur: Wolf B. Oerter)

\section{KRZYSZTOF OŻÓG}

\section{První generace profesorů na krakovské univerzitě v 15. století}

\section{RESUMÉ}

Krakovská univerzita byla založena dvakrát, roku 1364 a 1400. Organizátoři a profesoři první univerzity v Krakově, činní v letech 1364-1370, nejsou známi. Univerzita založená Vladislavem Jagiełłem roku 1400 byla organizována polskými a českými učenci, kteří vystudovali pražskou univerzitu. Největší migrační vlna z Prahy do Krakova proběhla v prvních letech 15. století. Celkem přišlo do roku 1415 do Krakova 54 graduovaných. Představovali první generaci krakovských profesorů na všech čtyřech fakultách. Druhá generace se zformovala díky mnohaleté činnosti mistrů, kteří přišli do Krakova z Prahy a z jiných evropských univerzit. Tyto dvě generace profesorů ovlivnily rozhodujícím způsobem vývoj krakovské univerzity, která patřila v 15. století mezi nejvýznamnější intelektuální centra ve střední a východní Evropě.

(český překlad Blanka Zilynská)

Prof. dr hab. Krzysztof Ożóg

https://orcid.org/0000-0001-6771-7813

Uniwersytet Jagielloński, Instytut Historii

krzysztof.ozog@uj.edu.pl 